\title{
ON THE GENERATORS OF THE SYMPLECTIC MODULAR GROUP
}

\author{
BY \\ L. K. HUA AND I. REINER
}

Introduction. Let $n$ be a positive integer. Throughout this paper, unless the contrary is stated, we shall use capital Latin letters to denote $n$-rowed matrices and capital German letters to denote $2 n$-rowed matrices. Furthermore, an $r$-rowed matrix $R$ will be denoted by $R^{(r)}$. Let

$$
\mathfrak{F}=\left(\begin{array}{rr}
0 & I \\
-I & 0
\end{array}\right)
$$

where $I$ and 0 denote the identity and zero matrices respectively. Let $\Gamma$ be the group of all matrices $\mathfrak{M}$ with rational integral elements which satisfy

$$
\mathfrak{M F M} \mathfrak{M}^{\prime}=\mathfrak{F}
$$

where $\mathfrak{M}^{\prime}$ denotes the transpose of $\mathfrak{M}$. Let $\Gamma_{0}$ be the factor group of $\Gamma$ over its centrum; $\Gamma_{0}$ is called the symplectic modular group. It can be thought of as being obtained from $\Gamma$ by identifying the elements $\mathfrak{M}$ and $-\mathfrak{M}$. In applications to modular functions of the $n$th degree $\left({ }^{1}\right)$ and to the projective geometry of matrices $\left({ }^{2}\right)$ it is customary to identify $\mathfrak{M}$ and $-\mathfrak{M}$ as a single transformation. For this reason we have considered $\Gamma_{0}$ rather than $\Gamma$; it might be pointed out, however, that the generators of $\Gamma_{0}$ obtained in this paper happen to be a set of generators of $\Gamma$.

It is the aim of this paper to find the generators of the symplectic modular group. It will be proved here that this group is generated by two or four independent elements, according as $n=1$ or $n>1$. The method used here can be extended so as to give a set of generators for matrices with elements in any Euclidean ring. In particular, we give the details for the generalized Picard group at the end of this paper.

Problems of this type have been considered previously. Poincaré( $\left.{ }^{3}\right)$ stated without proof that every matrix $\mathfrak{M}$ with integral elements for which $\mathfrak{M}(s \mathfrak{M}$ $=(\$)$, where $(\$)$ is the direct sum of $n$ two-rowed skew-symmetric matrices, is expressible as a product of elementary matrices of two simple types. Brahana $\left({ }^{4}\right)$ proved this and extended the result to the case where $(f)$ is any skewsymmetric matrix by showing in this case that every such matrix $\mathfrak{M}$ is ex-

Presented to the Society, February 28, 1948; received by the editors March 6, 1948.

(1) C. L. Siegel, Math. Ann. vol. 116 (1939) pp. 617-657.

(2) L. K. Hua, Trans. Amer. Math. Soc. vol. 57 (1945) pp. 441-490.

(3) H. Poincaré, Rend. Circ. Mat. Palermo vol. 18 (1904) pp. 45-110.

(4) H. R. Brahana, Ann. of Math. (2) vol. 24 (1923) pp. 265-270. 
pressible as a product of matrices taken from some finite set of matrices. From the results given in the present paper, a much stronger form of Brahana's result can be easily deduced.

1. If we set

$$
\mathfrak{M}=\left(\begin{array}{ll}
A & B \\
C & D
\end{array}\right),
$$

(1) is equivalent to

$$
A B^{\prime}=B A^{\prime}, \quad C D^{\prime}=D C^{\prime}, \quad A D^{\prime}-B C^{\prime}=I .
$$

By taking inverses of both sides of (1) and using $\mathfrak{F}^{-1}=-\mathfrak{F}$, we can deduce that $\mathfrak{M} \mathfrak{F} \mathfrak{M}=\mathfrak{F}$, so that

$$
A^{\prime} C=C^{\prime} A, \quad B^{\prime} D=D^{\prime} B, \quad A^{\prime} D-C^{\prime} B=I .
$$

We shall begin by showing in $\$ 3$ that $\Gamma_{0}$ is generated by the following types of elements:

(I) Translations:

$$
\mathfrak{I}=\left(\begin{array}{ll}
I & S \\
0 & I
\end{array}\right)
$$

where $S$ is symmetric.

(II) Rotations:

$$
\Re=\left(\begin{array}{ll}
U & 0 \\
0 & U^{\prime-1}
\end{array}\right),
$$

where $U$ is unimodular, that is, abs $U=1$ (where abs $U$ denotes the absolute value of the determinant of $U$ ).

(III) Semi-involutions:

$$
\Im=\left(\begin{array}{cc}
J & I-J \\
J-I & J
\end{array}\right)
$$

where $J$ is a diagonal matrix whose diagonal elements are 0 's and 1 's, so that $J^{2}=J$ and $(I-J)^{2}=I-J$.

It is easily verified that matrices of types I, II and III satisfy (1).

2. In this section we prove two lemmas on matrices.

LEMMA 1. Let $m$ be a nonzero integer, and let $T$ be an n-rowed symmetric matrix at least one of whose elements is not divisible by $m$. There exists a symmetric matrix $S$ with integral elements such that

$$
0<\operatorname{abs}(T-m S)<|m|^{n} \text {. }
$$

Proof. The lemma is evident for $n=1$. Consider next $n=2$; let $T=\left(t_{i j}\right)$, $S=\left(s_{i j}\right)$. Then 


$$
\text { abs }(T-m S)=\left|\left(t_{11}-m s_{11}\right)\left(t_{22}-m s_{22}\right)-\left(t_{12}-m s_{12}\right)^{2}\right| .
$$

If $m$ divides both $t_{11}$ and $t_{22}$, it cannot divide $t_{12}$; we can then choose $S$ so that $t_{11}-m s_{11}=t_{22}-m s_{22}=0$ and $0<\left|t_{12}-m s_{12}\right|<|m|$. Suppose on the other hand that $m$ does not divide one of the diagonal elements, say $t_{11}$. Fix $s_{12}$ arbitrarily, and choose $s_{11}$ so that $0<\left|t_{11}-m s_{11}\right|<|m|$. Since (6) can be written as

$$
\text { abs }(T-m S)=\left|-m\left(t_{11}-m s_{11}\right) s_{22}+\cdots\right| \text {, }
$$

where $\cdots$ represents terms not involving $s_{22}$, we can choose an integer $s_{22}$ by the Euclidean algorithm so that

$$
0<\operatorname{abs}(T-m S) \leqq\left|m\left(t_{11}-m s_{11}\right)\right|<|m|^{2} .
$$

Suppose now that the result has been established for $n=r-1$ with $r \geqq 3$; we shall deduce it for $n=r$. Let $T=T^{(r)}$ and let some element $t_{i j}$ of $T$ be not divisible by $m$. Since $r \geqq 3$, there exists a diagonal element $t_{k k}$ of $T$ which is not in the same row or column as $t_{i j}$. Let $T_{1}$ be the symmetric $(r-1)$-rowed matrix obtained from $T$ by omitting the $k$ th row and $k$ th column; let $S_{1}$ be similarly related to $S$. By the induction hypothesis, we may choose $S_{1}$ symmetric so that

$$
0<\operatorname{abs}\left(T_{1}-m S_{1}\right)<|m|^{r-1}
$$

However, we have

$$
\operatorname{abs}(T-m S)=\left|\left(t_{k k}-m s_{k k}\right) \operatorname{det}\left(T_{1}-m S_{1}\right)+\cdots\right|,
$$

where $\cdots$ represents terms not involving $s_{k k}$. Choose $s_{l k}$ arbitrarily for $l=1,2, \cdots, k-1, k+1, \cdots, r$. Then by the Euclidean algorithm we can choose $s_{k k}$ so that

$$
0<\operatorname{abs}(T-m S) \leqq|m| \text { abs }\left(T_{1}-m S_{1}\right)<|m| r .
$$

This completes the proof of the lemma.

Lemma 2. Let $A$ and $B$ satisfy $A B^{\prime}=B A^{\prime}$ and let $\operatorname{det} A \neq 0$. There exists a symmetric matrix $S$ such that either

$$
B-A S=0
$$

or

$$
0<\operatorname{abs}(B-A S)<\operatorname{abs} A \text {. }
$$

Proof. From $A B^{\prime}=B A^{\prime}$ and $\operatorname{det} A \neq 0$, we may deduce that $A^{*} B$ is symmetric, where $A^{*}$ denotes the adjoint of $A$. We apply Lemma 1 with $T=A^{*} B$ and $m=\operatorname{det} A$. Either every element of $A^{*} B$ is divisible by $m$, in which case there exists a symmetric matrix $S$ with $A^{*} B=m S$, or else there exist symmetric matrices $R$ and $S$ such that $A^{*} B=m S+R$ with $0<$ abs $R<|m|^{n}$. In virtue of the relation $A^{*} A=m I$, these alternatives become: either $B=A S$ (in 
which case (9) holds), or $B-A S=A R / m$; however,

$$
\text { abs } \frac{A R}{m}=\frac{(\operatorname{abs} A)(\operatorname{abs} R)}{|m|^{n}}=\frac{\text { abs } R}{|m|^{n-1}},
$$

so that

$$
0<\operatorname{abs}(B-A S)<|m|=\operatorname{abs} A \text {. }
$$

3. We are now ready to show that $\Gamma_{0}$ is generated by matrices of types $I$, II and III. Let $\mathfrak{M}$ given by (2) be an arbitrary element of $\Gamma_{0}$. It suffices t.o prove that by multiplying $\mathfrak{M}$ by matrices of types I, II and III, one obtains a product of matrices of those types.

(3) implies that not both $A$ and $B$ are 0 . Since

$$
\left(\begin{array}{ll}
A & B \\
C & D
\end{array}\right)\left(\begin{array}{rr}
0 & I \\
-I & 0
\end{array}\right)=\left(\begin{array}{rr}
-B & A \\
* & *
\end{array}\right)
$$

we may assume that $A$ has rank $r>0$. Furthermore,

$$
\left(\begin{array}{ll}
U & 0 \\
0 & U^{\prime-1}
\end{array}\right)\left(\begin{array}{ll}
A & B \\
C & D
\end{array}\right)\left(\begin{array}{ll}
V & 0 \\
0 & V^{\prime-1}
\end{array}\right)=\left(\begin{array}{cc}
U A V & * \\
* & *
\end{array}\right)
$$

so that $A$ may be taken to be of the form

$$
A=\left(\begin{array}{ll}
A_{1} & 0 \\
A_{2} & 0
\end{array}\right)
$$

where $A_{1}$ is an $r$-rowed nonsingular matrix. We similarly decompose $B$ as

$$
B=\left(\begin{array}{cc}
B_{1}^{(r)} & * \\
* & *
\end{array}\right)
$$

From (3) it is easily seen that $A_{1} B_{1}^{\prime}=B_{1} A_{1}^{\prime}$. By Lemma 2, there exists a symmetric matrix $S_{1}$ with either $A_{1} S_{1}+B_{1}=0$ or $0<$ abs $R_{1}<$ abs $A_{1}$, where $R_{1}=A_{1} S_{1}+B_{1}$. Define

$$
S=\left(\begin{array}{cc}
S_{1}^{(r)} & 0 \\
0 & 0
\end{array}\right)
$$

Then

$$
\left(\begin{array}{ll}
A & B \\
C & D
\end{array}\right)\left(\begin{array}{ll}
I & S \\
0 & I
\end{array}\right)=\left(\begin{array}{cc}
A & A S+B \\
* & *
\end{array}\right),
$$

so that $A$ remains unaltered while $B_{1}$ of $B$ is replaced by 0 or $R_{1}$. If the sec- 
ond alternative occurs, we proceed as follows: let

$$
J=\left(\begin{array}{ll}
0 & 0 \\
0 & I^{(n-r)}
\end{array}\right)
$$

Then

$$
\left(\begin{array}{ll}
A & B \\
C & D
\end{array}\right)\left(\begin{array}{cc}
J & I-J \\
J-I & J
\end{array}\right)=\left(\begin{array}{cc}
\bar{A} & * \\
* & *
\end{array}\right)
$$

where

$$
\bar{A}=A J-B(I-J)=\left(\begin{array}{cc}
-R_{1} & 0 \\
* & 0
\end{array}\right)
$$

We now repeat the process as before, and so on. Since there are only finitely many positive integers less than abs $A_{1}$, this process eventually terminates. Thus, by multiplying $\mathfrak{M}$ by matrices of types I, II and III one arrives at a matrix

$$
\left(\begin{array}{cc}
A_{0} & B_{0} \\
* & *
\end{array}\right)
$$

with

$$
A_{0}=\left(\begin{array}{ll}
R & 0 \\
* & 0
\end{array}\right), \quad B_{0}=\left(\begin{array}{ll}
0 & * \\
* & *
\end{array}\right)
$$

and det $R \neq 0$. One readily deduces from $A_{0} B_{0}^{\prime}=\dot{B}_{0} A_{0}^{\prime}$ that $B_{0}$ must be of the form

$$
B_{0}=\left(\begin{array}{ll}
0 & * \\
0 & *
\end{array}\right)
$$

But then

$$
\left(\begin{array}{rr}
0 & I \\
-I & 0
\end{array}\right)\left(\begin{array}{ll}
A_{0} & B_{0} \\
C_{0} & D_{0}
\end{array}\right)\left(\begin{array}{cc}
J & I-J \\
J-I & J
\end{array}\right)=\left(\begin{array}{cc}
A^{+} & B^{+} \\
0 & D^{+}
\end{array}\right)
$$

where $J$ is given by (13). Finally we notice that for a matrix

$$
\left(\begin{array}{ll}
A & B \\
0 & D
\end{array}\right)
$$

of $\Gamma_{0}$, we must have $A=U$ unimodular, $D=U^{\prime-1}$, and thence from (3), $B=S U^{\prime-1}$ with symmetric $S$. Therefore

$$
\left(\begin{array}{ll}
A & B \\
0 & D
\end{array}\right)=\left(\begin{array}{ll}
I & S \\
0 & I
\end{array}\right)\left(\begin{array}{ll}
U & 0 \\
0 & U^{\prime-1}
\end{array}\right)
$$


This completes the proof that $\Gamma_{0}$ is generated by the matrices of types I, II and III.

4. The set of matrices of types I, II and III which generate $\Gamma_{0}$ are certainly not independent generators. Let us reduce the number of generators as much as possible. Since

$$
\left(\begin{array}{cc}
I & S_{1} \\
0 & I
\end{array}\right)\left(\begin{array}{cc}
I & S_{2} \\
0 & I
\end{array}\right)=\left(\begin{array}{cc}
I & S_{1}+S_{2} \\
0 & I
\end{array}\right)
$$

the subgroup formed by matrices of type $I$ is generated by those type $I$ matrices whose $S$ 's are given by

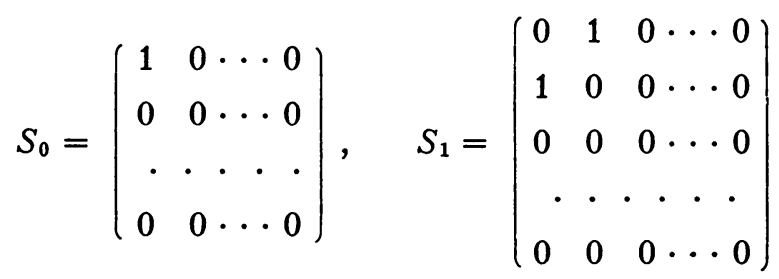

and all matrices obtained from these by interchanging any two rows and the corresponding columns. Next we note that

$$
\left(\begin{array}{ll}
U & 0 \\
0 & U^{\prime-1}
\end{array}\right)\left(\begin{array}{ll}
I & S \\
0 & I
\end{array}\right)\left(\begin{array}{cc}
U^{-1} & 0 \\
0 & U^{\prime}
\end{array}\right)=\left(\begin{array}{cc}
I & U S U^{\prime} \\
0 & I
\end{array}\right)
$$

so that the group generated by matrices of types I and II is the same as that generated by all type II matrices and the two translations whose $S$ 's are given by (15). However, we have

$$
\left(\begin{array}{rr}
1 & -1 \\
0 & 1
\end{array}\right)\left(\begin{array}{rr}
1 & 0 \\
0 & -1
\end{array}\right)\left(\begin{array}{rr}
1 & 0 \\
-1 & 1
\end{array}\right)=\left(\begin{array}{rr}
0 & 1 \\
1 & -1
\end{array}\right)=\left(\begin{array}{ll}
0 & 1 \\
1 & 0
\end{array}\right)-\left(\begin{array}{ll}
0 & 0 \\
0 & 1
\end{array}\right) .
$$

Hence the translation with $S_{1}$ is obtainable from that with $S_{0}$ and the matrices of type II. Therefore $\Gamma_{0}$ is generated by the matrix

$$
\mathfrak{T}_{0}=\left(\begin{array}{cc}
I & S_{0} \\
0 & I
\end{array}\right)
$$

with $S_{0}$ given by (15), and all matrices of types II and III.

Since

$$
\left(\begin{array}{ll}
U & 0 \\
0 & U^{\prime-1}
\end{array}\right)\left(\begin{array}{ll}
V & 0 \\
0 & V^{\prime-1}
\end{array}\right)=\left(\begin{array}{lc}
U V & 0 \\
0 & (U V)^{\prime-1}
\end{array}\right),
$$

in order to find the generators of the subgroup of rotations we have merely to find the generators of the group of unimodular matrices. These are given by the following theorem. 
THEOREM 1. Let $n \geqq 2$. Every unimodular matrix with rational integral elements is a product of the matrices $U_{1}, U_{2}, U_{3}$ and their inverses, where

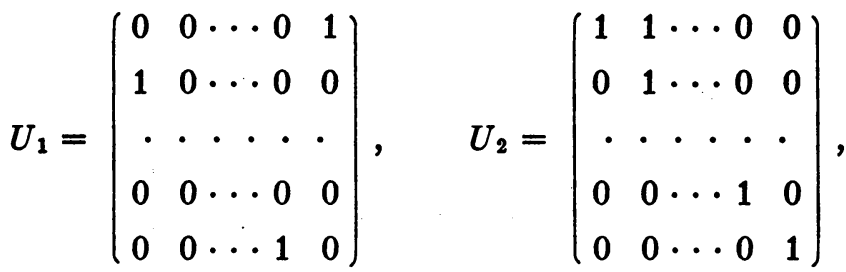

$$
\begin{aligned}
& U_{3}=\left(\begin{array}{rrrrr}
-1 & 0 & \cdots & 0 & 0 \\
0 & 1 & \cdots & 0 & 0 \\
\cdots & \cdot & \cdots & . & \cdot \\
0 & 0 & \cdots & 1 & 0 \\
0 & 0 & \cdots & 0 & 1
\end{array}\right)
\end{aligned}
$$

Proof. It is known $\left(^{5}\right)$ that every unimodular matrix is a product of $U_{1}, U_{2}, U_{3}$, and

$$
U_{4}=\left(\begin{array}{ccccc}
0 & 1 & \cdots & 0 & 0 \\
1 & 0 & \cdots & 0 & 0 \\
\cdot & \cdot & \cdot & . & \cdot \\
0 & 0 & \cdots & 1 & 0 \\
0 & 0 & \cdots & 0 & 1
\end{array}\right),
$$

and their inverses. It is sufficient to show that $U_{4}$ is expressible as a product of $U_{1}, U_{2}, U_{3}$ and their inverses. We define $T=U_{2} U_{1}$ for the remainder of this proof, and let $\mathfrak{r}=\left(r_{1}, \cdots, r_{n}\right)^{\prime}$ be a column vector. Then

$$
T \mathfrak{r}=\left(\begin{array}{c}
r_{n}+r_{1} \\
r_{1} \\
\vdots \\
r_{n-1}
\end{array}\right),
$$

$$
T^{2} \mathfrak{r}=\left(\begin{array}{r}
r_{n-1}+r_{n}+r_{1} \\
r_{n}+r_{1} \\
r_{1} \\
r_{n-2}
\end{array}\right), \cdots, T^{n-1} \mathfrak{r}=\left(\begin{array}{r}
r_{2}+r_{3}+\cdots+r_{n}+r_{1} \\
r_{3}+\cdots+r_{n}+r_{1} \\
\vdots \\
r_{n}+r_{1} \\
r_{1}
\end{array}\right) \text {. }
$$
22.5.

(5) See for example, C. C. MacDuffee, The theory of matrices, Berlin, 1933, p. 34, Theorem 
Therefore

$$
U_{1}^{-1} T^{n-1} \mathfrak{r}=\left(\begin{array}{r}
r_{3}+\cdots+r_{n}+r_{1} \\
\vdots \\
r_{n}+r_{1} \\
r_{1} \\
r_{2}+r_{3}+\cdots+r_{n}+r_{1}
\end{array}\right),
$$

so that

$$
\begin{gathered}
\left(T^{-1}\right)^{n-2} U_{1}^{-1} T^{n-1} \mathrm{r}= \\
\cdot\left(\begin{array}{c}
r_{1} \\
r_{2}+\cdots+r_{n}+r_{1} \\
r_{8} \\
r_{n}
\end{array}\right), \\
U_{1}\left(T^{-1}\right)^{n-2} U_{1}^{-1} T^{n-1} \mathfrak{r}=\left(\begin{array}{c}
r_{n} \\
r_{1} \\
r_{2}+r_{n}+r_{1} \\
r_{8} \\
\vdots \\
r_{n-1}
\end{array}\right) .
\end{gathered}
$$

From this we see that

$$
T^{n-8} U_{1}\left(T^{-1}\right)^{n-2} U_{1}^{-1} T^{n-1} \mathfrak{r}=\left(\begin{array}{r}
r_{3}+r_{4}+\cdots+r_{n} \\
r_{4}+\cdots+r_{n} \\
\vdots \\
r_{n} \\
r_{1} \\
r_{2}+\cdots+r_{n}+r_{1}
\end{array}\right)
$$

and

$$
\left(T^{-1}\right)^{n-2} U_{1} T^{n-2} U_{1}\left(T^{-1}\right)^{n-2} U_{1}^{-1} T^{n-1} \mathfrak{r}=\left(\begin{array}{c}
r_{n} \\
r_{1} \\
r_{2}+r_{1} \\
\vdots \\
r_{n-1}
\end{array}\right)
$$


Define

$$
U \dagger=U_{1}^{-1}\left(T^{-1}\right)^{n-2} U_{1} T^{n-3} U_{1}\left(T^{-1}\right)^{n-2} U_{1}^{-1} T^{n-1}
$$

Then

$$
U \dagger=\left(\begin{array}{ll}
1 & 0 \\
1 & 1
\end{array}\right)+I^{(n-2)},
$$

where $\dot{+}$ denotes the direct sum of matrices. But from

$$
U_{3}=\left(\begin{array}{rr}
-1 & 0 \\
0 & 1
\end{array}\right)+I^{(n-2)} \text { and } U_{2}^{-1}=\left(\begin{array}{rr}
1 & -1 \\
0 & 1
\end{array}\right)+I^{(n-2)}
$$

we deduce

$$
\begin{aligned}
U_{3} U \dagger U_{2}^{-1} U \dagger & =\left(\begin{array}{rr}
-1 & 0 \\
0 & 1
\end{array}\right)\left(\begin{array}{ll}
1 & 0 \\
1 & 1
\end{array}\right)\left(\begin{array}{rr}
1 & -1 \\
0 & 1
\end{array}\right)\left(\begin{array}{ll}
1 & 0 \\
1 & 1
\end{array}\right)+I^{(n-2)} \\
& =\left(\begin{array}{ll}
0 & 1 \\
1 & 0
\end{array}\right)+I^{(n-2)}=U_{4} .
\end{aligned}
$$

This completes the proof of the theorem.

COROLLARY. Let $n \geqq 2$. Every unimodular matrix with rational integral elements of determinant +1 is a product of powers of $U_{2}$ and

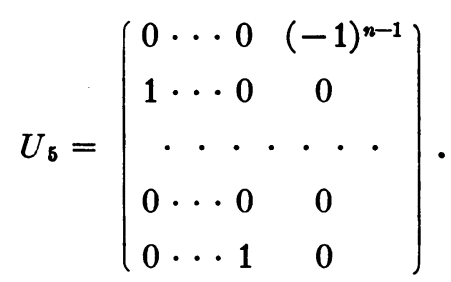

By Theorem 1 we see now that $\Gamma_{0}$ is generated by $\mathfrak{T}_{0}$ and the set of all semi-involutions and the three rotations defined by

$$
\Re_{i}=\left(\begin{array}{cc}
U_{i} & 0 \\
0 & U_{i}^{\prime-1}
\end{array}\right), \quad i=1,2,3 .
$$

We finally consider type III matrices. Let $J_{r}$ be the diagonal matrix obtained from the identity matrix by replacing the $r$ th 1 by 0 . In that case, if $r \neq s$, we have

$$
\left(\begin{array}{cc}
J_{r} & I-J_{r} \\
J_{r}-I & J_{r}
\end{array}\right)\left(\begin{array}{cc}
J_{s} & I-J_{s} \\
J_{s}-I & J_{s}
\end{array}\right)=\left(\begin{array}{cc}
J_{r s} & I-J_{r s} \\
J_{r s}-I & J_{r s}
\end{array}\right),
$$

where $J_{r s}$ is obtained from the identity matrix by replacing the $r$ th and $s$ th ones by 0's. Therefore, in order to obtain all type III matrices, we need only 
those semi-involutions

$$
\left(\begin{array}{cc}
J_{r} & I-J_{r} \\
J_{r}-I & J_{r}
\end{array}\right), . \quad r=1,2, \cdots, n,
$$

with $J_{r}$ defined above. Now, let $U$ be that unimodular matrix obtained from I by interchanging the 1 st and $r$ th rows; then we have

$$
\left(\begin{array}{ll}
U & 0 \\
0 & U^{\prime-1}
\end{array}\right)\left(\begin{array}{cc}
J_{r} & I-J_{r} \\
J_{r}-I & J_{r}
\end{array}\right)\left(\begin{array}{cc}
U^{-1} & 0 \\
0 & U^{\prime}
\end{array}\right)=\left(\begin{array}{cc}
J_{1} & I-J_{1} \\
J_{1}-I & J_{1}
\end{array}\right) .
$$

Therefore $\Gamma_{0}$ is generated by the matrices $\mathfrak{I}_{0}, \Re_{i}(i=1,2,3)$ and the matrix

$$
\Im_{0}=\left(\begin{array}{cc}
J_{1} & I-J_{1} \\
J_{1}-I & J_{1}
\end{array}\right)
$$

with $J$, previously defined. But

$$
\mathfrak{S}_{0}^{2}=\Re_{3},
$$

so that $\Re_{3}$ may be dropped from the list of generators. Therefore we have the following theorem.

THEOREM 2. $\Gamma_{0}$ is generated by the four matrices $\mathfrak{T}_{0}, \mathfrak{R}_{1}, \mathfrak{R}_{2}$ and $\mathfrak{S}_{0}$ given by (15), (18) and (20), for $n>1$. For $n=1, \Gamma_{0}$ is generated by $\mathfrak{T}_{0}$ and $\mathfrak{S}_{0}$.

5. In this section we shall prove the independence of the generators given in Theorem 2. For $n=1$, this is trivial because $\mathfrak{S}_{0}$ is of finite order while $\mathfrak{I}_{0}$ is not. Hereafter we suppose that $n>1$.

(1) Independence of $\mathfrak{T}_{0}$. We consider the transformation

$$
\left(X_{1}, Y_{1}\right)=(X, Y) \mathfrak{M} ;
$$

if $X Y^{\prime}$ is symmetric, it is easily verified that $X_{1} Y_{1}^{\prime}$ is also symmetric. We shall show that if the diagonal elements of $X Y^{\prime}$ are even, those of $X_{1} Y_{1}^{\prime}$ will also be even if $\mathfrak{M}$ is $\mathfrak{R}_{1}, \mathfrak{R}_{2}$ or $\mathfrak{S}_{0}$, while if $\mathfrak{M}=\mathfrak{T}_{0}$, it is possible to choose $X$ and $Y$ so that some diagonal element of $X_{1} Y_{1}^{\prime}$ is odd. This will show that $\mathfrak{T}_{0}$ is not expressible as a product of $\Re_{1}, \Re_{2}$ and $\mathfrak{S}_{0}$ and their inverses.

Assume now that the diagonal elements of $X Y^{\prime}$ are even. From (21) one readily deduces that if $\mathfrak{M}$ is a rotation, $X_{1} Y_{1}^{\prime}=X Y^{\prime}$, so that the diagonal elements of $X_{1} Y_{1}^{\prime}$ are also even. If secondly $\mathfrak{M}$ is a semi-involution, we have

$$
X_{1}=X J+Y(I-J), \quad Y_{1}=-X(I-J)+Y J,
$$

so that

$$
X_{1} Y_{1}^{\prime}=X J Y^{\prime}-Y(I-J) X^{\prime}=X J Y^{\prime}+Y J X^{\prime}-Y X^{\prime} .
$$

Since $X J Y^{\prime}$ is the transpose of $Y J X^{\prime}$, it is again clear that the diagonal ele- 
ments of $X_{1} Y_{1}^{\prime}$ are even. Finally, suppose $\mathfrak{M}=\mathfrak{T}_{0}$. Then we obtain

$$
X_{1} Y_{1}^{\prime}=X\left(X S_{0}+Y\right)^{\prime}=X Y^{\prime}+X S_{0} X^{\prime}
$$

and for $X=I$ the first diagonal element of $X_{1} Y_{1}^{\prime}$ is odd. This completes the proof of the independence of $\mathfrak{I}_{0}$. We may remark in passing, however, that $\mathfrak{T}_{0}^{2}$ is expressible as a product of the powers of $\Re_{1}, \Re_{2}$ and $\mathfrak{S}_{0}$.

(2) Independence of $\Re_{1}$. Let $\mathfrak{r}=\left(r_{1}, \cdots, r_{n}, s_{1}, \cdots, s_{n}\right)^{\prime}$ be a column vector with $2 n$ components. It is clear that the second component $r_{2}$ is unaffected when $\mathfrak{r}$ is multiplied on the left by any of the matrices $\mathfrak{I}_{0}, \Re_{2}$, and $\mathfrak{S}_{0}$ and their inverses. Under multiplication on the left by $\Re_{1}$, however, $r_{2}$ is replaced by $r_{1}$. Hence $\Re_{1}$ cannot be expressed as a product of $\mathfrak{T}_{0}, \Re_{2}$ and $\mathfrak{S}_{0}$ and their inverses.

(3) Independence of $\Re_{2}$. Multiplying $\mathfrak{r}$ on the left by $\Re_{1}$ or $\mathfrak{S}_{0}$ or their inverses permutes components of $\mathfrak{r}$; under any such permutation, however, any $r_{i}$ and its corresponding $s_{i}$ remain $n$ components apart. Since the effect of multiplying on the left by $\mathfrak{I}_{0}$ is to replace $r_{1}$ by $r_{1}+s_{1}$, it is clear that by multiplying $\mathfrak{r}$ on the left by a product of $\Re_{1}, \mathfrak{S}_{0}$ and $\mathfrak{I}_{0}$ and their inverses, $r_{1}$ may be replaced by a linear combination of $r_{1}$ and $s_{1}$ and its position may be changed. It is however impossible to replace $r_{1}$ by $r_{1}+r_{2}$ in this way, and this is exactly the effect of multiplication of $\mathfrak{r}$ on the left by $\Re_{2}$. This proves the independence of $\Re_{2}$.

(4) Independence of $\mathfrak{S}_{0}$. We note that

$$
\left(\begin{array}{ll}
* & * \\
0 & *
\end{array}\right)\left(\begin{array}{ll}
* & * \\
0 & *
\end{array}\right)=\left(\begin{array}{ll}
* & * \\
0 & *
\end{array}\right)
$$

Since $\mathfrak{T}_{0}, \Re_{1}$ and $\Re_{2}$ and their inverses are all of the form

$$
\left(\begin{array}{ll}
* & * \\
0 & *
\end{array}\right)
$$

and $\mathfrak{S}_{0}$ is not of this form, it is clear that $\mathfrak{S}_{0}$ is not expressible as a product of $\mathfrak{I}_{0}, \Re_{1}$ and $\Re_{2}$ and their inverses.

6. Our previous method can be extended to any Euclidean ring; in particular, for the ring formed by the Gaussian integers, we have the following result:

THEOREM 3. Let $\Gamma^{\prime}$ be the group of matrices $\mathfrak{M}$ with Gaussian integers as elements which satisfy (1). Let $\Gamma_{0}^{\prime}$ be obtained from $\Gamma^{\prime}$ by identifying the four elements $\pm \mathfrak{M}$ and $\pm i \mathfrak{M}$. Then for $n>1, \Gamma_{0}^{\prime}$ is generated by the matrices $\mathfrak{T}_{0}, \Re_{1}, \Re_{2}$ and $\mathfrak{S}_{0}$ defined previously, and the matrix

$$
\mathfrak{T}_{1}=\left(\begin{array}{ll}
I & S_{1} \\
0 & I
\end{array}\right) \quad \text { where } S_{1}=i S_{0} .
$$

For $n=1, \Gamma_{0}^{\prime}$ is generated by $\mathfrak{I}_{0}, \mathfrak{T}_{1}$ and $\mathfrak{S}_{0}$. 
The independence of the generators is shown as follows (with suitable modifications when $n=1$ ):

(1) Independence of $\mathfrak{T}_{0}$. We use the method of $\$ 5$, (1). Let $X Y^{\prime}$ be a symmetric matrix with Gaussian integers as elements, such that the real part of each diagonal element is even. This property is preserved when $(X, Y)$ is subjected to the transformations $\mathfrak{I}_{1}, \Re_{1}, \Re_{2}$ and $\mathfrak{S}_{0}$ according to $(21)$, but not for the transformation $\mathfrak{I}_{0}$.

(2) Independence of $\mathfrak{T}_{1}$. This is clear since $\mathfrak{T}_{1}$ is the only generator which is not real.

(3) The independence of $\Re_{1}, \Re_{2}$ and $\mathfrak{S}_{0}$ follow exactly as before.

\section{Tsing Hua University,}

Peiping, China.

INSTITUTE FOR ADVANCED STUdY,

Princeton, N. J. 